\title{
ORIENTACCÃO PARA O MERCADO EXTERNO: 0 REFINAMENTO DE UMA ESCALA DE MENSURAÇÃO
}

RESUMO

0 presente estudo consiste em um survey exploratório de corte transversal único, realizado com 617 empresas exportadoras brasileiras, desenvolvido com o objetivo de validar, no Brasil, uma escala de orientação para o mercado externo. Utilizou-se a modelagem de equações estruturais como base para a análise estatística dos dados coletados. Duas escalas de mensuração de orientação para o mercado serviram de referência para o estudo - a escala de orientação para o mercado externo de Cadogan, Diamantopoulos e Mortanges (1999) e a escala de orientação para o mercado de Matsuno, Mentzer e Rentz (2000b). A escala resultante - um modelo híbrido, unindo indicadores das duas escalas citadas, mas principalmente alicerçada sobre o modelo de Cadogan et al. (1999) - demonstrou resultados confiáveis e apresenta-se como um modelo válido para mensurar o grau de orientação para o mercado externo das organizações exportadoras brasileiras.

\section{Ivan Lapuente Garrido}

UNISINOS

\begin{abstract}
ABSIRACTThe present study is an exploratory survey of a transversal cut, carried through with 617 Brazilian exporting companies, developed with the objective to validate, in Brazil, a scale of export market orientation. Structural equation modeling was used as the base for the statistical analysis statistics of the collected data. Two scales of measuring of market orientation had served of reference for the study - the scale of export market orientation developed by Cadogan, Diamantopoulos, and Mortanges (1999) and the scale of market orientation created by Matsuno, M entzer, and Rentz (2000b). The resulting scale - a hybrid model, joining indicators of the two scales mentioned, but mainly based on the model by Cadogan et al. (1999) - demonstrated trustworthy results and is presented as a valid model to measure the degree of orientation for the external market of Brazilian exporting organizations.
\end{abstract}

PALAMRAS-CHAVE Orientação para o mercado, orientação para o mercado externo, internacionalização, ambiente internacional, marketing internacional.

KEMORDS $M$ arket orientation, export market orientation, internationalization, international environment, international marketing. 


\section{INTRODUÇÃO}

No Brasil, os estudos mais significativos, na tentativa de validar uma escala de orientação para o mercado externo, foram os de Macera e U rdan (2004) e o de Garrido, Larentis e Slongo (2006). Em ambos os estudos, entretanto, foram encontradas limitações quanto à validade e confiabilidade dos resultados.

Com o intuito de fornecer um instrumento quepermita captar de forma mais confiável a orientação para o mercado externo entre as empresas brasileiras exportadoras, este estudo percorreu três etapas distintas: primeiramente, tentou validar a escala de Cadogan, Diamantopoulos e Mortanges (1999); em seguida, adaptou a escala de Matsuno, M entzer e Rentz (2000b) para 0 ambiente internacional e testou-a entre empresas exportadoras brasileiras; e, por fim, testou um model o híbrido que contempla indicadores das duas escal as citadas. Foram pesquisadas 617 empresas exportadoras brasileiras.

Este estudo inicia com um breve referencial teórico abordando o tema orientação para mercado externo e as escalas de Cadogan, Diamantopoulos e Mortanges (1999) e de Matsuno, Mentzer e Rentz (2000b). Em seguida, apresenta o estudo empírico realizado e discute seus resultados.

\section{REFERENCIAL TEÓRICO}

\section{Orientação para o mercado externo}

Na década de 1990, ressurgiu o interesse prático e acadêmico em orientação para mercado. Entretanto, apesar da relevante importância das operações internacionais para a sobrevivência das organizações, os estudos concentraram seu interesse nos mercados domésticos (Diamantopoulos e Cadogan, 1996).

Mesmo que as pesquisas em internacionalização já estudassem diversos determinantes do comportamento de marketing para exportação, a adaptação do constructo de orientação para o mercado e para 0 ambiente internacional e a verificação da possível relação com performance internacional passaram a ser exploradas somente por volta do início dos anos 2000, tendo como principais expoentes os estudos de Cadogan e Diamantopoulos (1995); Diamantopoulos e Cadogan (1996); Cadogan, Diamantopoulos e Mortanges (1999); e Cadogan, Diamantopoulos e Siguaw (2002).

Com base nos dois conceitos dominantes de orientação para o mercado - Kohli e Jaworski (1990) e Narver e Slater (1990) -, Cadogan e Diamantopoulos (1995) pro- põem um modelo de adaptação do modelo de orientação para o mercado ao ambiente internacional. 0 modelo é composto por quatro construtos - coordenação interfuncional, geração de intel igência exportadora, disseminação de inteligência exportadora e ação de resposta.

A geração, disseminação e ação de resposta à intel igência de mercado, realizadas durante todo o processo por meio da coordenação interfuncional, refletem-se orientadas tanto ao consumidor quanto aos concorrentes. 0 mecanismo de coordenação interfuncional garante que as atividades genéricas associadas à orientação para o mercado sejam realizadas de forma efetiva e eficaz (Cadogan e Diamantopoulos, 1995). Ele inclui o elemento da dependência interfuncional de Narver e Slater (1990), integrado com o elemento de responsabilidade partilhada por toda a organização, de Kohli e Jaworski (1990), e a adição de uma dimensão suplementar às atividades interfuncionais, a da dinâmica das interações entre membros, pois as funções individuais afetam a habilidade da organização em criar valor superior para os compradores-alvos (Cadogan e Diamantopoulos, 1995).

Os componentes de orientação para mercado permanecem conceitualmente os mesmos, entretanto a operacional ização dos construtos, quando focados em um ambiente internacional, implicam modificações nos itens de medida existentes, bem como a inclusão de novos fatores, uma vez que as empresas se expõem a forças ambientais diferentes e mais complexas que aquelas enfrentadas no mercado doméstico. Forças legais, políticas, econômicas, competitivas, tecnológicas e socioculturais, tanto quanto logísticas e geográficas, influem na implementação do conceito de marketing das empresas.

\section{A mensuração de orientação para o mercado externo}

Cadogan, Diamantopoulos e M ortanges (1999) desenvolveram e validaram empiricamente um instrumento para mensurar o grau de orientação para o mercado externo e a relação com performance em negócios internacionais. Seguiram a perspectiva de Cadogan e Diamantopoulos (1995), os quais propõem um modelo integrativo dos estudos de Kohli e Jaworski (1990) e de Narver e Slater (1990) adaptado ao ambiente internacional.

0 construto de orientação para o mercado externo proposto por Cadogan, Diamantopoulos e M ortanges (1999) consiste em três componentes comportamentais ( geração de inteligência exportadora, com 11 itens; disseminação da inteligência exportadora, com 18 itens; ação de resposta, com 17 itens, todos orientados para competidores e consumidores) e em uma dimensão integrativa (me- 
canismo de coordenação, com 25 itens). Cada item foi medido por meio de uma escala do tipo Likert. A escala foi testada com uma amostra de empresas da Alemanha e do Reino Unido, a fim de confirmar a validade entre diferentes culturas.

Posteriormente, a escala foi utilizada por Cadogan, Diamantopoulos e Siguaw (2002) e Akyol e Akehurst (2003). No Brasil, a escala de Cadogan, Diamantopoulos e Mortanges (1999) foi testada por Macera e Urdan (2004) e por Garrido, Larentis e Slongo (2006). Macera e Urdan (2004) encontraram validade discriminante apenas parcialmente satisfatória para os construtos de geração de inteligência e disseminação de inteligência, e relação positiva parcial entre orientação para o mercado e performance exportadora. Dentre as observações dos autores, encontram-se a possibilidade de a amostragem ser pequena e a necessidade de aprimoramento do modelo de orientação para o mercado externo, seja pela elaboração de uma nova escala, seja pelo refinamento da escal a de Cadogan, Diamantopoulos e M ortanges (1999). Garrido, Larentis e Slongo (2006) obtiveram resultados parcial mente satisfatórios. Os autores encontraram al guns problemas de val idades convergente e discriminante e de confiabilidade dos construtos. A pontaram, como prováveis causadores dessa parcialidade, a extensão da escala, o elevado número de questões reversas e a semelhança entre al gumas questões.

\section{A escala de orientação para o mercado de Matsuno, Mentzer e Rentz}

Matzuno, M entzer e Rentz (2000b) indicam que a questão da rel ação entre orientação para o mercado e performance, apesar de já bastante investigada, não foi bem analisada focando diferentes estratégias. Assim, examinam empiricamente o papel dos tipos de estratégia de negócio como potenciais moderadores da relação orientação para mercado - performance.

A lógica central de Matsuno, M entzer e Rentz (2000b) é que a implementação de uma estratégia em particular é essencialmente um processo de adaptação organizacional ao ambiente de mercado, no qual a orientação para o mercado tem um papel fundamental.

Para esse estudo, M atsuno, M entzer e Rentz (2000b) aprimoraram as propriedades da escala M arkor, de Kohli, Jaworski e Kurmar (1993). A escala Markor, segundo Matsuno et al. (2000b), foca o grupo de comportamentos usados pela firma para responder a aspectos críticos de mercado - consumidores, competidores, aspectos legais, forças societais e macroeconômicas. Os autores dizem que, apesar de toda a teoria existente que conduzi ria à inclusão de múltiplos stakeholders na operacionalização de uma escala de orientação para mercado, a escala M arkor foca principalmente o domínio dos consumidores e dos competidores e não explicita como outros fatores de mercado, por exemplo 0 ambiente legal e regulatório e 0 ambiente macroeconômico, podem influenciar a competição e os consumidores.

\section{METODOLOGIA}

Este estudo pode ser classificado como um survey exploratório de corte transversal único (Malhotra, 2004).

Três escalas de orientação para o mercado externo foram testadas neste estudo. A escal a proposta por Cadogan et al. (1999). Essa escala foi selecionada por ser o estudo mais significativo sobre o tema e por já ter sido utilizada em diferentes países, inclusive no Brasil. A escala de Matsuno, Mentzer e Rentz (2000b), selecionada em função da preocupação de seus autores em relacionar 0 tema com estratégias organizacionais e considerarem os elementos do ambiente externo como grandes influenciadores das decisões organizacionais.

Por fim, em função dos resultados parcialmente satisfatórios, decidiu-se pela retomada da escala de Cadogan et al. (1999), com o acréscimo de algumas alterações, motivadas pelas análises baseadas no primeiro teste da escala - tais como a modificação de algumas questões reversas; a adequação de termos técnicos de comércio exterior; a exclusão de questões com conteúdos muito semelhantes. Ao mesmo tempo, foram incluídos alguns indicadores da escala de Matsuno et al. (2000b) que se mostraram significativos.

\section{Amostra e coleta dos dados}

As empresas exportadoras brasileiras representam a população-alvo desta pesquisa. 0 Brasil conta com cerca de 16 mil empresas cadastradas como exportadoras. Foi usado, como base para o contato com as empresas, o cadastro dos importadores/exportadores brasileiros fornecido pela Confederação N acional das Indústrias (CNI). A amostra foi do tipo não probabilístico (Malhotra, 2004), extraída por conveniência do referido cadastro. Os dados referentes às empresas exportadoras brasileiras estão à disposi ção no site http://www.brazilexport4.com.

Para a efetivação da pesquisa foi necessária a coleta de dados em três momentos distintos. A primeira col eta, que se orientou pela meta de validação da escala de Cadogan et al. (1999), ocorreu nos meses de novembro e dezembro de 2005. Foram contatadas, por telefone, 600 empresas 
brasileiras exportadoras, para verificar sua disponibilidade em responder aos questionários da pesquisa. Dessas, 332 responderam ao questionário.

A segunda coleta de dados ocorreu no mês de junho de 2006 e teve como objetivo a tentativa de validação da escala adaptada do estudo de Matsuno, M entzer e Rentz (2000b). Foram entrevistados membros de 66 empresas brasileiras exportadoras. Por fim, a terceira coleta de dados, buscando a validação da escala híbrida, pesquisou 219 empresas exportadoras brasileiras e ocorreu durante 0 mês de agosto de 2006.

A técnica de coleta de dados baseou-se em contatos telefônicos com as empresas. Na prática, sete pesquisadores foram treinados e acompanhados durante o processo das entrevistas nas três etapas anteriormente descritas. Esse método foi escolhido por diversas razões: primeira, a necessidade de o tempo de coleta ser pequeno, de modo a reproduzir as memas condições do ambiente externo em que estão expostos os respondentes; segunda, a facilidade de resposta, já que os questionários, por serem grandes, poderiam desestimular a resposta espontânea; eterceira, 0 melhor controle dos filtros, de modo a selecionarmos apenas os executivos de marketing internacional, responsáveis pela estratégia de internacionalização das empresas.

Foram, ainda, realizadas diversas entrevistas com acadêmicos de marketing internacional e com executivos de comércio exterior para a análise qual itativa das escalas empregadas. Para a condução dessas entrevistas, utilizaramse as próprias escal as, bem como os resultados estatísticos parciais encontrados em cada fase.

A adaptação dos instrumentos de coleta de dados ocorreu em duas etapas. Primeiro, as escalas originais foram traduzidas para a língua portuguesa por um especialista; em seguida, entrevistas em profundidade foram real izadas com executivos e acadêmicos de comércio exterior para ajuste dos termos específicos, compreensão das questões, análise qualitativa dos instrumentos, acessibilidade e comodidade para os respondentes.

Utilizou-se uma escala do tipo Likert com seis pontos (de '1 - Discordo totalmente' a ' 6 - Concordo totalmente') para captar a opinião dos respondentes em rel ação aos indicadores. Uma escala desse tipo, quando se considera a soma geral dos indicadores, pode ser tratada como uma escala intervalar, possibilitando o uso de multivariadas técnicas de análise estatística (Nunnally e Berstein, 1995).

\section{Procedimentos estatísticos para a análise dos dados}

A análise quantitativa dos dados contemplou etapas de tratamento dos dados e de análises estatísticas por meio do uso de modelagem de equações estruturais.
Para Hair Jr. et al. (2005), antes de aplicar qualquer técnica multivariada, o pesquisador deve avaliar 0 ajuste dos dados da amostra com as suposições estatísticas da técnica multivariada a ser usada na análise dos dados. Foi verificada a existência de respondentes que apresentavam padrões de resposta muito diferentes dos demais (outliers) (Bagozzi, 1994; Kline, 1998). Essa verificação foi realizada por meio de avaliação multivariada. Nesse caso, segundo Hair Jr. et al. (2005), a medida D2 de Mahalanobis (uma medida da distância, em um espaço multidimensional, de cada observação em relação ao centro médio das observações, resultando em uma medida de centralidade multidimensional) pode ser usada para esse propósito. Os autores sugerem que um nível de significância de 0,001 seja utilizado como base para determinar uma observação atípica. Com relação a dados omissos, Kline (1998) destaca que não devam ser superiores a uma faixa de 5 a $10 \%$ das respostas de uma variável, acrescentando, ainda, que essa omissão deva ser al eatória.

A normalidade dos dados foi observada por meio da verificação dos val ores de assimetria e curtose, conforme sugerido por Hair Jr. et al. (2005). Kline (1988) aponta que variáveis com valores absolutos de índices de assimetria acima de |3| podem ser muito assimétricas e com valores de curtose acima de (10) podem ser problemáticas para a normalidade dos dados.

A homoscedasticidade, segundo Hair Jr. et al. (2005), refere-se à suposição de que as variáveis dependentes exibem níveis iguais de variância ao longo do domínio das variáveis preditoras. A homoscedasticidade e a linearidade foram verificadas por meio dos diagramas de dispersão.

As análises estatísticas ocorreram com o uso do SPSS (Statistical Package for Social Sciences) para análises descritivas para o tratamento de dados e para análises de variância e de regressão múltipla. Foi usado o AMOS (Analysis of Moment Structures) para a modelagem de equações estruturais na validação e adaptação das escalas de orientação para mercado externo. Foi usada a estratégia de modelagem confirmatória. Ela representa o uso mais direto em modelagens de equações estruturais, por meio da avaliação de sua significância estatística (Hair J r. et al., 2005). Esta proposta é adequada, pois buscaramse a adaptação e a validação de uma escala de orientação para mercado externo. 0 método de avaliação utilizado foi o de máxima verossimilhança e a matriz de entrada de dados foi a de covariância. Esses métodos, al ém de serem os padrões do programa AM OS, são os mais utilizados no uso de modelagem de equações estruturais ( Hair J r. et al., 
2005; Kline, 1998). Cada um dos construtos foi anal isado separadamente, e, por fim, o modelo como um todo, conforme recomenda Bagozzi (1994).

Kline (1998) sugere que, mesmo que não haja uma resposta única ao modelo testado, deve-se proceder à adequação do modelo proposto. A qualidade do ajuste mede a correspondência da matriz de dados de entrada observados com aquelas previstas pelo modelo proposto (Hair Jr. et al., 2005). Existem diversas medidas de ajustamento. Hair Jr. et al. (2005), por exemplo, as classificam de três formas: a) medidas de ajuste absoluto: avaliam apenas o ajuste geral do modelo, determinam o grau em que o modelo geral prediz a matriz de covariância ou correlação; b) medidas de ajuste incremental: comparam o modelo proposto com um modelo nulo; c) medidas de ajuste parcimonioso: acertam as medidas de ajuste para fornecer uma comparação entre model os com diferentes números de coeficientes estimados.

Os principais índices de estimação utilizados são: Quiquadrado sobre Graus de Liberdade ( $\sigma^{2} / G L$ ); Goodnessof-Fit (GFI); Root Mean Square Error of Approximation (RMSEA); Adjusted Goodness-of-Fit Index (AGFI); Turcker-Lewis Index (TLI); Normed Fit Index (NFI), Comparative Fit Index (CFI).

Após o ajuste geral do modelo, a mensuração de cada constructo pode ser avaliada quanto à unidimensionalidade e à confiabilidade. A unidimensionalidade é demonstrada quando os indicadores de um construto têm ajuste aceitável sobre um modelo de um só fator. 0 uso de medidas de confiabilidade, como o alfa de Cronbach, não garante a unidimensionalidade, mas assume que ela existe (Hair Jr. et al., 2005). 0 alfa de Cronbach foi estimado para os construtos analisados.

A confiabilidade representa uma medida de consistência interna dos indicadores de um constructo descrevendo o grau em que estes indicam o construto latente. Foi calculada a confiabilidade de cada construto separadamente. Um valor de referência comumente usado para confiabilidade aceitável é 0,7 , apesar de valores abaixo deste serem aceitáveis em pesquisas de natureza exploratória (Hair Jr. et al., 2005). A variância extraída representa uma medida de confiabilidade: indica a quantidade geral de variância nos indicadores explicada pelo construto latente. Foi cal culada a variância extraída para cada um dos construtos separadamente. Sugeremse valores superiores a 0,5 para um construto (Hair Jr. et al., 2005).

A validade convergente de cada um dos construtos foi verificada em função das cargas fatoriais resultantes, dos resíduos padronizados abaixo de I2,58I (Garver e M entzer,
1999), da confiabilidade composta (acima de 0,70 ) e da variância extraída (acima de 0,50) (Hair Jr. et al., 2005). $A$ validade discriminante entre os construtos foi verificada a partir do que recomendam Fornell e Larcker (1981), ou seja, a variância extraída de cada construto deve ser maior que as variâncias compartilhadas (correlação ao quadrado) entre os construtos.

\section{RESULTADOS}

\section{A escala de Cadogan, Diamantopoulos e Mortanges (1999)}

As entrevistas qualitativas, realizadas com acadêmicos e executivos, apontaram como pontos negativos da escala a sua extensão, com 71 questões e a aparente semelhança entre várias delas. Também apontaram a existência de muitas questões reversas que poderiam confundir os respondentes. N os construtos de orientação para o mercado externo pôde-se observar que as questões estão divididas em três blocos, abordando os temas orientação para consumidor, orientação para competidor e questões de natureza ambígua, que podem ser orientadas tanto para concorrentes quanto para clientes, já que apresentam foco geral no mercado.

Mesmo com diversas informações advindas da análise qualitativa, optou-se, neste momento, por não excluir qualquer questão da escala antes da aplicação empírica em empresas exportadoras brasileiras. Nesse sentido, a análise qual itativa serviu como subsídio para futuras modificações na escala.

Após a adaptação qualitativa do instrumento de mensuração de orientação para mercado externo de Cadogan et al. (1999), procedeu-se a sua aplicação em empresas exportadoras brasileiras. N esta etapa, 332 empresas participaram da pesquisa. A preparação dos dados seguiu os padrões descritos no método de pesquisa. Foram eliminados 19 casos, resultando em uma amostra final de 313 casos. Dentro da model agem de equações estruturais ( $M E E)$, para validação de escalas, foi feita a análise fatorial confirmatória (AFC).

Apresentam-se, a seguir (Tabela 1 ), os resultados encontrados para cada um dos construtos de orientação para mercado externo e, em seguida, os respectivos comentários.

\section{Geração de inteligência exportadora}

No modelo inicial de Cadogan et al. (1999), a geração de inteligência exportadora era definida por 11 itens. O modelo final, após os testes necessários, resultou em 
um construto com cinco itens. Verifica-se, com base nos resultados, que o constructo está com valores do TLI e do $\chi 2$ /GL muito próximos do recomendado; em relação ao RMSEA, está um pouco acima do recomendado; à confiabilidade composta, está um pou co abaixo, e, por fim, quanto à variância extraída, está abaixo do recomendado.

\section{Disseminação de inteligência exportadora}

0 modelo inicial baseado em Cadogan et al. (1999) continha 18 itens. 0 model o final adaptado resultou em quatro itens. Verifica-se que o constructo está com valores do RMSEA um pouco abaixo do recomendado; da confiabilidade composta e da variância extraída, abaixo do recomendado. Os demais índices estão de acordo com 0 recomendado pela literatura.

\section{Ação de resposta}

0 modelo inicial continha 17 questões; por sua vez, o modelo final adaptado resultou em seis questões. Verifica-se que o constructo está com valores do RMSEA um pouco acima do recomendado e da variância extraída um pouco abaixo do recomendado. Os demais índices estão de acordo com o recomendado pela literatura.

\section{Coordenação interfuncional}

0 modelo inicial continha 25 questões. De acordo com as entrevistas real izadas com executivos e acadêmicos para a análise qual itativa da escala, essa extensão foi indicada como muito grande. Dessa forma, após os testes, resultaram nove questões. Verifica-se que o constructo está com valores do AGFI muito próximos do recomendado, do RMSEA um pouco acima do recomendado e da variância extraída um pouco abaixo do recomendado. Os demais índices atendem aos padrões recomendados.

\section{Validade convergente, discriminante e multicolinearidade}

Verificou-se, a partir da análise individual de cada um dos construtos, que el es apresentaram, de forma geral, validade convergente, em função das cargas fatoriais resultantes elevadas (acima de 0,50 ), resíduos padronizados abaixo de I2,58I (Garver e M entzer, 1999), confiabilidade composta acima de 0,70 (com exceção dos construtos geração e disseminação de inteligência), tendo como maior limitação a baixa variância extraída encontrada, já que somente 0 construto coordenação interfuncional ficou bem próximo a 0,50.

Para a validade discriminante entre os construtos, 0 teste de Fornell e Larcker (1981) recomenda que a variância extraída de cada construto deve ser maior que as variâncias compartilhadas entre os construtos ( correlações ao quadrado). N esse sentido, verificam-se variâncias compartilhadas maiores em rel ação às variâncias extraídas (ou seja, falta de validade discriminante) entre os construtos geração de inteligência e ação de resposta, disseminação de inteligência e ação de resposta, disseminação de inteligência e coordenação interfuncional e ação de resposta e coordenação interfuncional (Tabela 2).

A partir da análise da matriz de correlação (Tabela 2), não se identifica a existência de multicolinearidade, já que todos os construtos apresentaram intercorrelações abaixo de 0,85, conforme sugere Kline (1998). Dessa forma, a escal a proposta, levando em consideração tanto as entrevistas qualitativas quanto a análise quantitativa, demonstra adequação apenas parcial da escala.

Como aspectos positivos, obteve-se redução de 71 para 24 questões. Como pontos negativos, a val idação da escala de orientação para mercado externo apresentou limitações na confiabilidade composta, na variância extraída e na val idade discriminante. Esses resultados foram conse-

Tabela 1 - Análise fatorial confirmatória dos construtos de orientação para o mercado externo.

\begin{tabular}{|c|c|c|c|c|c|c|c|c|c|c|c|c|}
\hline CONSTRUTO & $x^{2}$ & $P$ & GL & $\begin{array}{c}\chi^{2 /} \\
G L\end{array}$ & GFI & AGFI & $\mathrm{NFI}$ & TLI & $\mathrm{CFI}$ & RMSEA & $\begin{array}{l}\text { CONF. } \\
\text { COMP. }\end{array}$ & $\begin{array}{l}\text { VAR. } \\
\text { EXTR. }\end{array}$ \\
\hline $\begin{array}{l}\text { Geração de } \\
\text { inteligência }\end{array}$ & 25,48 & 0,00 & 5 & 5,094 & 0,967 & 0,900 & 0,935 & 0,890 & 0,946 & 0,115 & 0,59 & 0,23 \\
\hline $\begin{array}{l}\text { Disseminação de } \\
\text { inteligência }\end{array}$ & 2,92 & 0,23 & 2 & 1,460 & 0,995 & 0,976 & 0,988 & 0,989 & 0,996 & 0,038 & 0,53 & 0,23 \\
\hline Ação de resposta & 31,66 & 0,00 & 9 & 3,520 & 0,966 & 0,922 & 0,954 & 0,943 & 0,966 & 0,09 & 0,79 & 0,39 \\
\hline $\begin{array}{l}\text { Coordenação } \\
\text { interfuncional }\end{array}$ & 105,73 & 0,00 & 27 & 3,520 & 0,935 & 0,892 & 0,925 & 0,924 & 0,943 & 0,097 & 0,89 & 0,47 \\
\hline Padrões & & & & $<5$ & $>0,8$ & $>0,8$ & $>0,9$ & $>0,9$ & $>0,8$ & 0,05 a 0,08 & $>0,7$ & $>0,5$ \\
\hline
\end{tabular}


qüências, principalmente, dos erros elevados associados a diversas variáveis. Isso não permitiu que se obtivesse a validade convergente adequada (uma vez que a confiabilidade composta e a variância extraída, entre outras análises, atestam isso).

Verificou-se também falta de validade discriminante entre al guns construtos, uma vez que a variância extraída faz parte da sua anál ise, em comparação com as variâncias compartilhadas (correlações ao quadrado). Parte dessas limitações talvez se justifique nas análises qualitativas realizadas. Provavelmente a extensão da escala e o elevado número de questões inversas (neste caso, a maioria não permaneceu na escala final) tenham conduzido a el evados erros associados às medidas detectados durante a análise dos dados.

\section{A escala de orientação para o mercado de Matsuno, Mentzer e Rentz}

Após a adaptação qualitativa do instrumento por meio das entrevistas com executivos e acadêmicos, procedeu- se a sua aplicação em empresas exportadoras brasileiras. Foram selecionadas 66 empresas brasileiras exportadoras. O ptou-se por se fazer um teste com uma amostra pequena de empresas para primeiro assegurar que os resultados seriam superiores aos encontrados para a escala de Cadogan et al. (1999). Se isso ocorresse, proceder-se-ia a uma nova coleta de dados - mas, neste caso, com uma amostra superior.

Da mesma forma que nos testes realizados para tentar validar a escala de Cadogan et al. (1999), os dados foram preparados conforme caracterizado no método de pesquisa. Foram eliminados seis questionários. Dessa forma, a amostra final resultou em 60 casos.

\section{Análise fatorial confirmatória}

A presentam-se, a seguir, os resultados da análise fatorial confirmatória encontrados para cada um dos construtos de orientação para mercado externo. A Tabela 3 apresenta os resultados estatísticos encontrados, seguidos dos respectivos comentários.

Tabela 2 - Análise discriminante e multicolinearidade.

\begin{tabular}{|l|c|c|c|c|}
\hline \multirow{2}{*}{ CONSTRUTOS } & \multicolumn{3}{|c|}{ VARIÂNCIA EXTRAIDA E VARIÂNCIAS } \\
\cline { 2 - 5 } & $\begin{array}{c}\text { GEM PAR TILHADAS } \\
\text { INTEL. }\end{array}$ & $\begin{array}{c}\text { DISSEM. } \\
\text { INTEL. }\end{array}$ & $\begin{array}{c}\text { AÇÃO } \\
\text { RESP. }\end{array}$ & $\begin{array}{l}\text { COORD. } \\
\text { INTERF. }\end{array}$ \\
\hline $\begin{array}{l}\text { Geração de } \\
\text { inteligência }\end{array}$ & 0,23 & & \\
\hline $\begin{array}{l}\text { Disseminação de } \\
\text { inteligência }\end{array}$ & 0,19 & 0,23 & \\
\hline $\begin{array}{l}\text { Ação de resposta } \\
\text { Coordenação }\end{array}$ & 0,37 & 0,28 & 0,39 & \\
\hline interfuncional & 0,23 & 0,29 & 0,48 & 0,47 \\
\hline
\end{tabular}

\begin{tabular}{|l|c|c|c|c|}
\hline \multirow{2}{*}{ CONS TRUTOS } & \multicolumn{4}{|c|}{ MATRIZ DE CORRELAÇÃO } \\
\cline { 2 - 5 } & $\begin{array}{c}\text { GER. } \\
\text { INTEL. }\end{array}$ & $\begin{array}{c}\text { DISSEM. } \\
\text { INTEL. }\end{array}$ & $\begin{array}{c}\text { AÇÃO } \\
\text { RESP. }\end{array}$ & $\begin{array}{l}\text { COORD. } \\
\text { INTERF. }\end{array}$ \\
\hline $\begin{array}{l}\text { Geração de } \\
\text { inteligência }\end{array}$ & 1,00 & & \\
\hline $\begin{array}{l}\text { Disseminação de } \\
\text { inteligência }\end{array}$ & 0,44 & 1,00 & \\
\hline $\begin{array}{l}\text { Ação de resposta } \\
\text { Coordenação }\end{array}$ & 0,61 & 0,53 & 1,00 & \\
\hline interfuncional & 0,48 & 0,53 & 0,70 & 1,00 \\
\hline
\end{tabular}

Tabela 3 - Análise fatorial confirmatória da escala de Matsuno, Mentzer e Rentz.

\begin{tabular}{|c|c|c|c|c|c|c|c|c|c|c|c|c|}
\hline CONSTRUTO & $x^{2}$ & $P$ & $\mathrm{GL}$ & $\begin{array}{c}\chi^{2 /} \\
G L\end{array}$ & GFI & AGFI & NFI & TLI & CFI & RMSEA & $\begin{array}{l}\text { CONF. } \\
\text { COMP. }\end{array}$ & $\begin{array}{l}\text { VAR. } \\
\text { EXTR. }\end{array}$ \\
\hline $\begin{array}{l}\text { Geração de } \\
\text { inteligência }\end{array}$ & 2,751 & 0,25 & 2 & 1,58 & 0,968 & 0,905 & 0,924 & 0,966 & 0,977 & 0,924 & 0,646 & 0,383 \\
\hline $\begin{array}{l}\text { Disseminação de } \\
\text { inteligência }\end{array}$ & 0,236 & 0,88 & 2 & 0,12 & 0,997 & 0,992 & 0,993 & 1,08 & 1,0 & 0 & 0,59 & 0,33 \\
\hline Ação de resposta & 31,66 & 0,00 & 9 & 3,520 & 0,966 & 0,922 & 0,954 & 0,943 & 0,966 & 0,09 & 0,79 & 0,39 \\
\hline Padrões & & & & $<5$ & $>0,8$ & $>0,8$ & $>0,9$ & $>0,9$ & $>0,8$ & 0,05 a 0,08 & $>0,7$ & $>0,5$ \\
\hline
\end{tabular}


Geração de inteligência exportadora

No modelo inicial adaptado de Matsuno, Mentzer e Rentz (2000b), a geração de inteligência era definida por oito itens. 0 melhor modelo determinado, após os testes realizados, resultou em um construto com três itens, com as seguintes cargas fatoriasi: 0,691, 0,812 e 0,554.

Verifica-se que o construto está com valores de ajuste do modelo de acordo com os valores recomendados na literatura. Somente o valor do RMSEA está um pou co acima do recomendado. A confiabilidade composta está um pouco abaixo do indicado ea variância extraída encontrase consideravelmente abaixo do recomendado.

\section{Disseminação de inteligência exportadora}

No modelo inicial, o constructo disseminação de inteligência exportadora continha seis itens. 0 mel hor model 0 encontrado resultou em um construto com três itens, com as seguintes cargas fatoriais: $0,636,0,662$ e 0,72 . O bservase a inadequação do modelo evidenciada nos valores de TLI, CFI e RMSA, bem como na baixa confiabilidade composta e na variância extraída. Entretanto, três indicadores apresentaram cargas fatoriais elevadas.

\section{Ação de resposta}

O construto inicial para ação de resposta continha sete indicadores, todos eles mantidos após a análise fatorial confirmatória. A pesar de os índices de ajuste do modelo apresentarem-se rel ativamente adequados, a carga fatorial de cada um dos itens do construto ficou bastante baixa menor que 0,41 , com exceção apenas de um item, o qual apresentou carga de 0,604.

Os resultados obtidos no teste com a escala adaptada de M atsuno, M entzer e Rentz (2000b) não apresentaram resultados satisfatórios, seja pela inadequação dos índices de ajuste do model o, seja pelas baixas cargas fatoriais dos indicadores que compõem os construtos de orientação para mercado externo.

\section{A escala final: modelo híbrido de Cadogan etal. (1999) e Matsuno, Mentzer e Rentz (2000b)}

Em função das limitações detectadas com os resultados dos testes das duas escal as anteriores, optou-se por nova tentativa de validação de uma escala de orientação para o mercado externo mais confiável. Utilizou-se, como alicerce para esse novo teste, a escal a adaptada de Cadogan et al. (1999) agregando-se a ela os indicadores da escala adaptada de Matsuno, Mentzer e Rentz (2000b) que apresentaram carga fatorial el evada.

Procedeu-se, novamente, a uma série de entrevistas com três acadêmicos da área de marketing internacional e com três executivos de negócios internacionais para a avaliação e adaptação da nova escala proposta.

No construto de geração de inteligência foram mantidos todos os cinco itens da escala adaptada e testada de Cadogan et al. (1999) e, ainda, incluiu-se um item (3) que havia sido excluído no primeiro teste empírico do constructo. Essa inclusão deveu-se ao fato de esse item representar a orientação para o concorrente e por essa orientação não estar representada diretamente no construto resultante do primeiro teste empírico. N ovamente por indicação dos entrevistados, esse item, que originalmente solicitava resposta reversa, foi alterado para uma questão direta. Da escala adaptada de Matsuno et al. (2000b), foram incorporados ao construto dois itens que apresentaram carga fatorial el evada. Aos cinco itens da escala adaptada de Cadogan et al. (1999).

Para a disseminação de inteligência exportadora, foram acrescentados aos indicadores do teste da escala de Cadogan et al. (1999) três itens da escala adaptada de Matsuno et al. (2000b). E a questão 26, oriunda de Cadogan et al. (1999), que original mente era reversa, foi transformada em direta.

Para a determinação do construto de ação de resposta, os entrevistados sugeriram a reinclusão dos itens 35,39 e 40 da escala de Cadogan et al. (1999) por se tratarem claramente de itens que refletem respostas da empresa baseadas na inteligência gerada. Sugeriram também que fossem feitas algumas alterações no enunciado das questões: a transformação da questão 35 em questão direta e a substituição do termo "negócios externos" por "negócios internacionais" nas questões 39 e 40. Somente um item da escal a adaptada de M atsuno, M entzer e Rentz (2000b) apresentou elevada carga fatorial. Entretanto, como ele trata de orientação para fornecedor e essa orientação não condiz com o modelo conceitual de Cadogan et al. (1999), que contempla somente orientação para o consumidor e para o cliente, optou-se por não incluí-lo no constructo a ser testado.

Por fim, na anál ise qual itativa do construto coordenação interfuncional, os entrevistados analisaram as nove questões que compunham o construto da escala adaptada de Cadogan et al. (1999) e concordaram que as questões 69 e 70 eram repetitivas, por abordarem conteúdos já claramente expressos nas questões 47 e 48 . Como o constructo já apresentava al to número de questões - nove - e considerando-se esse caráter de sobreposição de conteúdo, optou-se pelo corte das questões 69 e 70.

A escala foi aplicada em uma amostra de 219 empresas brasileiras exportadoras. A preparação dos dados seguiu os padrões indicados no método da pesquisa: foram eli- 
minados 17 questionários, resultando em uma amostra de 202 questionários válidos.

\section{Análise fatorial confirmatória}

A presentam-se, a seguir (Tabela 4), os resultados da análise fatorial confirmatória encontrados para cada um dos construtos de orientação para o mercado externo.

\section{Geração de inteligência exportadora}

A nova escala utilizada apresenta, para o construto geração de inteligência exportadora, oito itens. Seis provenientes da escala de Cadogan et al. (1999) - G1, G2, G3, G4, G5 e $G 6$ - e dois itens provenientes da escala de Matsuno et al. (2000b) - G7 e G8.

0 modelo resultante, após os testes realizados, foi um constructo com cinco itens. Os itens G2, G 4 e G 7 estão relacionados à orientação para os consumidores; o G3, para os competidores; e o G1 é de natureza dúbia: tanto pode ser relativo a consumidores como a competidores.

Os índices encontrados demonstram o bom nível de ajuste do model o. Todos os itens apresentam-se dentro dos parâmetros estabel eci dos na literatura para a aceitação do model o, com exceção da variância extraída, com valor de 0,41 , ficando um pouco abaixo do valor recomendado de 0,5 (Hair Jr. et al., 2005) . 0 al fa de Cronbach encontrado para o constructo foi 0,822 , acima do 0,7 recomendado como valor mínimo por Hair Jr. et al. (2005).

Disseminação de inteligência exportadora

A escal a proposta continha oito itens para o construto disseminação de inteligência exportadora. D1, D2, D3, D4 e
D5, provenientes da escala de Cadogan et al. (1999), eD6, D7 e D8, de Matsuno, Mentzer e Rentz (2000b).

Após os testes estatísticos, o constructo resultou em 6 itens, três da escala de Cadogan et al. (1999) - D1, D3 e D4 - e três da escala adaptada de M atsuno, Mentzer e Rentz (2000b) - D6, D7 e D8. Os indicadores D6, D7 e D8 têm natureza dúbia quanto à orientação. Os indicadores D1 e D3 são orientados para competidores, ao passo que o D4 é orientado para consumidor.

O bserva-se que todos os índices encontrados atendem às indicações de adequação propostas na literatura estudada. Somente a variância extraída $(0,4)$ está pouco abaixo do recomendado (0,5). 0 alfa de Cronbach para o constructo resultante é 0,854. De acordo com Hair Jr. et al. (2005), o limite mínimo geralmente aceito para 0 alfa é 0,70 .

\section{Ação de resposta}

0 construto de ação de resposta proposto continha oito itens, todos provenientes da escala de Cadogan et al. (1999). Os indicadores R1, R2, R5 e R7 são orientados para os consumidores; $R 4$ é orientado para o competidor; e R8 é de natureza dúbia.

Assim como os construtos de geração de inteligência e disseminação de inteligência, os índices de ajuste estão de acordo com os padrões exigidos na literatura para indicarem a adequação de um modelo. Somente a variância extraída permaneceu pouco abaixo do indicado; porém, superior ao valor encontrado nos demais construtos. 0 alfa de Cronbach encontrado para os indicadores que compõem o construto de ação de resposta foi 0,859 , acima, portanto, do 0,7 sugerido por Hair Jr. et al. (2005).

Tabela 4 - Análise fatorial confirmatória dos construtos que compõem a orientação para o mercado externo.

\begin{tabular}{|c|c|c|c|c|c|c|c|c|c|c|c|c|}
\hline CONSTRUTO & $x^{2}$ & $P$ & $\mathrm{GL}$ & $\chi 2 / G L$ & GFI & AGFI & $\mathrm{NFI}$ & TLI & CFI & RMSEA & $\begin{array}{l}\text { CONF. } \\
\text { COMP. }\end{array}$ & $\begin{array}{l}\text { VAR. } \\
\text { EXTR. }\end{array}$ \\
\hline $\begin{array}{l}\text { Geração de } \\
\text { inteligência }\end{array}$ & 23,8 & 0,00 & 5 & 4,76 & 0,950 & 0,851 & 0,934 & 0,892 & 0,946 & 0,085 & 0,77 & 0,41 \\
\hline $\begin{array}{l}\text { Disseminação de } \\
\text { inteligência }\end{array}$ & 32,712 & 0,000 & 9 & 3,63 & 0,946 & 0,874 & 0,988 & 0,914 & 0,948 & 0,074 & 0,79 & 0,40 \\
\hline Ação de resposta & 52,47 & 0,000 & 14 & 3,75 & 0,930 & 0,860 & 0,904 & 0,900 & 0,927 & 0,084 & 0,85 & 0,45 \\
\hline $\begin{array}{l}\text { Coordenação } \\
\text { interfuncional }\end{array}$ & 38,47 & 0,000 & 14 & 2,75 & 0,948 & 0,896 & 0,958 & 0,959 & 0,973 & 0,059 & 0,93 & 0,64 \\
\hline $\begin{array}{l}\text { Orientação para o } \\
\text { mercado externo }\end{array}$ & 450,41 & 0,000 & 265 & 1,70 & 0,852 & 0,818 & 0,894 & 0,922 & 0,931 & 0,059 & & \\
\hline Padrões & & & & $<5$ & $>0,8$ & $>0,8$ & $>0,9$ & $>0,9$ & $>0,8$ & $\begin{array}{c}0,05 a \\
0,08\end{array}$ & $>0,7$ & $>0,5$ \\
\hline
\end{tabular}




\section{Coordenação interfuncional}

0 constructo de coordenação interfuncional foi originalmente estabelecido em sete indicadores. Após os testes realizados, todos os indicadores permaneceram no construto. Todos os índices encontrados satisfazem as condições recomendadas pela literatura para indicarem a adequação do construto. 0 alfa de Cronbach para 0 construto foi 0,92 , bem acima de 0,7 - recomendado por Hair Jr. et al. (2005).

Com base nos índices de ajuste encontrados na análise individual dos construtos que compõe a orientação para o mercado externo, verificou-se a adequação desses construtos, com exceção da variância extraída - que, para os construtos de geração de inteligência exportadora, disseminação da inteligência e ação de resposta ficou um pouco abaixo do recomendado. Entretanto, outros resultados, tais como a confiabilidade composta e 0 alfa de Cronbach, indicam a confiabilidade dos construtos. Os demais índices são satisfatórios.

A partir desses resultados, procedeu-seà verificação dos índices de ajustamento para o model o completo de orientação para o mercado externo. A Figura 1 demonstra 0 modelo sintético de orientação para o mercado externo.

Os valores dos índices deajustamento do modelo (Tabela 4) apresentam-se, de manei ra geral, dentro dos parâmetros recomendados. Somente o NFI apresenta-se levemente inferior ao índice recomendado. 0 alfa de Cronbach encontrado para os indicadores que compõem a orientação para o mercado externo foi 0,933. Em seguida, foram identificadas as cargas fatoriais pad ronizadas para verificar-se a validade das relações propostas no modelo. A Tabela 5 demonstra os parâmetros estimados do modelo.

Os dados das cargas fatoriais indicam que as relações propostas no modelo de orientação para o mercado exter-

Figura 1 - Modelo sintético de orientação para o mercado externo.

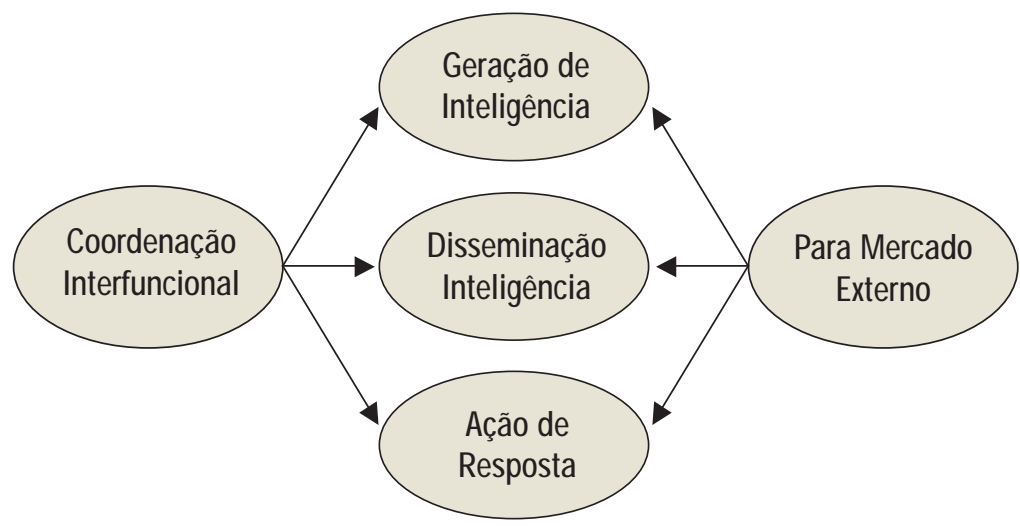

Fonte: Elaborado com base em Cadogan et al. (1999).

Tabela 5 - Análise fatorial confirmatória do modelo de orientação para o mercado externo.

\begin{tabular}{|c|c|c|}
\hline RELAÇÃO & $\begin{array}{c}\text { CARGA FATORIAL } \\
\text { PADRONIZADA }\end{array}$ & tvalue \\
\hline Geração de inteligência $\rightarrow$ Orientação para o mercado externo & 0,533 & $3,507 *$ \\
\hline Ação de resposta $\rightarrow$ Orientação para o mercado externo & 0,631 & $4,063^{*}$ \\
\hline Disseminação de inteligência $\rightarrow$ Orientação para o mercado externo & 0,440 & $3,631 *$ \\
\hline Ação de resposta $\rightarrow$ Coordenação interfuncional & 0,737 & $8,337 *$ \\
\hline Disseminação de inteligência $\rightarrow$ Coordenação interfuncional & 0,669 & $7,838 *$ \\
\hline Geração de inteligência $\rightarrow$ Coordenação interfuncional & 0,408 & $4,714^{*}$ \\
\hline
\end{tabular}

$* p<0,01$ 
no são significantes ao nível de 0,01. A validade convergente foi verificada em função das el evadas cargas fatoriais resultantes para cada um dos indicadores (Tabela 6), dos resíduos padronizados que ficaram abaixo de $|2,58|$, da confiabilidade composta acima de 0,70 e da variância extraída acima de 0,50.

A validade discriminante entre os construtos foi verificada a partir do teste de Fornell e Larcker (1981), que recomenda que a variância extraída de cada construto deve ser maior que as variâncias compartilhadas entre os construtos (correlações ao quadrado). A Tabela 7 apresenta a comparação entre a variância extraída de cada construto (diagonal) e as variâncias compartilhadas entre os construtos. Os resultados demonstram a validade discriminante entre os construtos. Quanto à multicolinearidade, a análise da matriz de correl ação (Tabela 7) demonstra que todos os construtos apresentaram intercorrel ações abaixo de 0,85 , conforme sugere Kline (1998), não demonstrando, assim, a existência de multicolinearidade.
Com base em todos os resultados obtidos na análise do modelo de orientação para o mercado externo (índices de ajustes, cargas fatoriais das relações, val idades convergentes e discriminantes), pode-se dizer que o modelo apresentado para a orientação para o mercado externo é válido. A escala testada pode ser observada no Quadro 1.

\section{CONSIDERAÇÕES FINAIS E IMPLICAÇÕES}

A partir da adaptação do modelo de orientação para o mercado externo de Cadogan et al. (1999) e da inclusão de indicadores provenientes do estudo de Matsuno, M entzer e Rentz (2000b), a pesquisa apresentou uma escala válida e confiável de orientação para o mercado externo, mais simplificada e com menor número de indicadores em cada construto.

Essas características possibilitarão o aprofundamento de estudos em internacionalização por facultarem o uso

Tabela 6 - Cargas fatoriais resultantes para os indicadores de orientação para o mercado externo.

\begin{tabular}{|c|c|c|c|c|c|c|c|}
\hline \multirow{2}{*}{$\begin{array}{l}\text { Indicadores de geração de inteligência } \\
\text { cargas fatoriais }\end{array}$} & G1 & G2 & G3 & G4 & G7 & & \\
\hline & 0,639 & 0,657 & 0,803 & 0,716 & 0,563 & & \\
\hline \multirow{2}{*}{$\begin{array}{l}\text { Indicadores de disseminação de inteligência } \\
\text { cargas fatoriais }\end{array}$} & D8 & D7 & D6 & D4 & D1 & D3 & \\
\hline & 0,68 & 0,694 & 0,663 & 0,607 & 0,714 & 0,77 & \\
\hline \multirow{2}{*}{$\begin{array}{l}\text { Indicadores de ação de resposta } \\
\text { cargas fatoriais }\end{array}$} & R1 & R2 & R3 & R4 & R5 & R7 & R8 \\
\hline & 0,683 & 0,674 & 0,689 & 0,735 & 0,724 & 0,611 & 0,626 \\
\hline \multirow{2}{*}{$\begin{array}{l}\text { Indicadores de coordenação interfuncional } \\
\text { cargas fatoriais }\end{array}$} & $\mathrm{Cl}$ & C2 & C3 & C4 & C5 & C6 & C7 \\
\hline & 0,829 & 0,862 & 0,748 & 0,789 & 0,825 & 0,763 & 0,707 \\
\hline
\end{tabular}

Tabela 7 - Análise discriminante e multicolinearidade.

\begin{tabular}{|c|c|c|c|c|c|c|c|c|c|}
\hline \multirow[b]{2}{*}{ CONSTRUTOS } & \multicolumn{4}{|c|}{$\begin{array}{l}\text { VARIÂNCIA EXTRAÍDA E VARIÂNCIAS } \\
\text { COMPARTILHADAS }\end{array}$} & \multirow[b]{2}{*}{ CONSTRUTOS } & \multicolumn{4}{|c|}{ MATRIZ DE CORRELAÇÃO } \\
\hline & $\begin{array}{l}\text { GER. } \\
\text { INTEL. }\end{array}$ & $\begin{array}{l}\text { DIS- } \\
\text { SEM. } \\
\text { INTEL. }\end{array}$ & $\begin{array}{l}\text { AÇÃO } \\
\text { RESP. }\end{array}$ & $\begin{array}{l}\text { COORD. } \\
\text { INTERF. }\end{array}$ & & $\begin{array}{l}\text { GER. } \\
\text { INTEL. }\end{array}$ & $\begin{array}{l}\text { DIS- } \\
\text { SEM. } \\
\text { INTEL. }\end{array}$ & $\begin{array}{l}\text { AÇÃO } \\
\text { RESP. }\end{array}$ & $\begin{array}{l}\text { COORD. } \\
\text { INTERF. }\end{array}$ \\
\hline $\begin{array}{l}\text { Geração de } \\
\text { inteligência }\end{array}$ & 0,41 & & & & $\begin{array}{l}\text { Geração de } \\
\text { inteligência }\end{array}$ & 1,00 & & & \\
\hline $\begin{array}{l}\text { Disseminação de } \\
\text { inteligência }\end{array}$ & 0,20 & 0,44 & & & $\begin{array}{l}\text { Disseminação de } \\
\text { inteligência }\end{array}$ & 0,45 & 1,00 & & \\
\hline Ação de resposta & 0,28 & 0,40 & 0,45 & & Ação de resposta & 0,53 & 0,67 & 1,00 & \\
\hline $\begin{array}{l}\text { Coordenação } \\
\text { interfuncional }\end{array}$ & 0,12 & 0,38 & 0,43 & 0,64 & $\begin{array}{l}\text { Coordenação } \\
\text { interfuncional }\end{array}$ & 0,34 & 0,62 & 0,66 & 1,00 \\
\hline
\end{tabular}


Quadro 1 - Escala híbrida de orientação para o mercado, adaptada de Cadogan, Diamantopoulos e Mortanges (1999) e Matsuno, Mentzer e Rentz (2000b).

GERAÇÃO DE INTELIGÊNCIA EXP
ITENS
G1. Nós geramos muitas informações a respeito de tendências nos nossos
mercados internacionais (ex. regulação, desenvolvimentos tecnológicos,
política, economia).
G2. Nós geramos muitas informações a fim de entender as forças
que influenciam as necessidades e preferências de nossos clientes
internacionais.

G3. Nós geramos muitas informações a fim de monitorar e entender a forma de atuação dos nossos concorrentes em mercados internacionais.

G4. Nós, constantemente, monitoramos nosso nível de comprometimento e orientação para atender às necessidades de nossos clientes no exterior

G5. Nossas altas gerências, de todos os departamentos funcionais (ex. marketing, produção, finanças) visitam regularmente nossos clientes internacionais.

G6. Nós medimos a satisfação dos clientes nos mercados externos de forma sistemática e regular.

G7. Nós, periodicamente, avaliamos o possível efeito das mudanças nos ambientes dos mercados doméstico e internacional sobre os nossos clientes do exterior (ex. normas, regulamentações, câmbio, barreiras, etc.).

G8. Nós, regularmente, coletamos e avaliamos informações macroeconômicas gerais (ex. taxas de juros, taxa de câmbio, PIB, taxa de crescimento industrial, taxa de inflação).
ORIGEM

Item 6 de Cadogan

et al. (1999)

Item 7 de Cadogan

et al. (1999)

Item 8 de Cadogan

et al. (1999)

Item 9 de Cadogan

et al. (1999)

Item 10 de Cadogan et al. (1999)

Item 11 de Cadogan et al. (1999)

Item 3 de Matsuno

et al. (2000b)

Item 4 de Matsuno et al. (2000b)
ORIENTAÇÃO

RESULTADO

Validado

Dúbia

Validado

Consumidores

Validado

Concorrentes

Validado

Consumidores

Validado

Consumidores

Não validado

Consumidores

Não validado

Consumidores

Validado

Dúbia

Não validado

\begin{tabular}{|c|c|c|c|}
\hline \multicolumn{4}{|c|}{ DISSEMINAÇÃO DE INTELIGÊNCIA EXPORTADORA } \\
\hline ITENS & ORIGEM & ORIENTAÇÃOO & RESULTADO \\
\hline $\begin{array}{l}\text { D1. Nós compartilhamos toda informação a respeito de nossos concor- } \\
\text { rentes dos mercados internacionais com todos os departamentos da } \\
\text { nossa empresa. }\end{array}$ & $\begin{array}{l}\text { Item } 14 \text { de Cado- } \\
\text { gan et al. (1999) }\end{array}$ & Competidores & Validado \\
\hline $\begin{array}{l}\text { D2. Informações que podem influenciar a maneira como atendemos } \\
\text { os nossos clientes internacionais chegam ao pessoal de exportação } \\
\text { rapidamente. }\end{array}$ & $\begin{array}{l}\text { Item } 15 \text { de Cado- } \\
\text { gan et al. (1999) }\end{array}$ & Consumidores & Não validado \\
\hline $\begin{array}{l}\text { D3. } 0 \text { pessoal de exportação regularmente compartilha informações com } \\
\text { toda a nossa empresa, a respeito das estratégias de nossos concorrentes } \\
\text { nos mercados internacionais. }\end{array}$ & $\begin{array}{l}\text { Item } 16 \text { de Cado- } \\
\text { gan et al. (1999) }\end{array}$ & Competidores & Validado \\
\hline $\begin{array}{l}\text { D4. Nós, livremente, trocamos informações sobre as experiências bem e } \\
\text { mal-sucedidas com nossos clientes internacionais, entre todos os depar- } \\
\text { tamentos funcionais da empresa. }\end{array}$ & $\begin{array}{l}\text { Item } 17 \text { de Cado- } \\
\text { gan et al. (1999) }\end{array}$ & Consumidores & Validado \\
\hline
\end{tabular}




\section{ARTIGOS ORIENTAÇÃO PARA O MERCADO EXTERNO: 0 REFINAMENTO DE UMA ESCALA DE MENSURAÇÃO}

(continuação)

D5. A alta gerência da nossa empresa, regularmente discute e avalia as for-

D6. Nós temos, freqüentemente, encontros interdepartamentais para discutirmos tendências e desenvolvimentos de mercados internacionais (ex. clientes, concorrência, fornecedores).

D7. Nós, regularmente, temos encontros interdepartamentais para atualizarmos nosso conhecimento a respeito de exigências e normas internacionais.

D8. 0 pessoal da área técnica, na nossa empresa, dedica muito tempo compartilhando informações sobre tecnologia para desenvolvimento de novos produtos com outros departamentos. ças e fraquezas dos nossos concorrentes dos mercados internacionais.
Item 18 de Cadogan et al. (1999)

Item 11 de Matsuno et al. (2000b)

Item 12 de Matsuno et al. (2000b)

Item 13 de Matsuno et al. (2000b)
Competidores

Não validado

AÇÃO DE RESPOSTA

\section{ITENS}

R1. Nós, periodicamente, revisamos nossos esforços de desenvolvimento de produtos, a fim de assegurar que eles estarão de acordo com o que nossos clientes internacionais querem.

R2. Nós damos muita importância aos serviços de pós-vendas em nossos mercados externos.

R3. Nossos objetivos para os negócios internacionais são determinados, principalmente, pelas necessidades e satisfação dos clientes.

R4. Nós respondemos rapidamente às ações competitivas dos nossos concorrentes que nos ameaçam em nossos mercados externos.

R5. Nossa estratégia para criação de vantagens competitivas nos mercados internacionais se baseia em nosso entendimento das necessidades dos clientes internacionais.

R6. Os produtos que vendemos no mercado internacional são determinados mais pelas necessidades dos clientes do que pelas políticas da empresa.

R7. Quando detectamos que os clientes internacionais estão insatisfeitos ou sugerem mudanças em nossos produtos ou serviços, nós tomamos ações corretivas imediatamente.

R8. Nós somos rápidos para responder às mudanças ambientais que podem afetar nossos negócios internacionais.

\section{ORIGEM}

Item 32 de Cadogan et al. (1999)

Item 43 de Cadogan et al. (1999)

Item 44 de Cadogan et al. (1999)

Item 45 de Cadogan et al. (1999)

Item 46 de Cadogan et al. (1999)

Item 35 de Cadogan et al. (1999)

Item 39 de Cadogan et al. (1999)

Item 40 de Cadogan et al. (1999)
ORIENTAÇÃO

Consumidores

Validado

Consumidores

Validado

Consumidores

Validado

Competidores

Validado

Consumidores

Validado

Consumidores

Não validado

Consumidores

Validado

Dúbia

Validado

\section{COORDENAÇÃO INTERFUNCIONAL}

\section{ITENS}

C1. Na nossa empresa todos os departamentos trabalham juntos, como um time, em relação aos nossos negócios de internacionais.

C2. As atividades dos diferentes departamentos em nossa empresa estão integradas e bem articuladas entre si na busca de uma meta em comum.

\begin{tabular}{|c|c|}
\hline ORIGEM & RESULTADO \\
\hline Item 47 de Cadogan et al. (1999) & Validado \\
\hline Item 48 de Cadogan et al. (1999) & Validado \\
\hline
\end{tabular}




\section{IVAN LAPUENTE GARRIDO}

(continuação)

C3. Na nossa empresa, quando ocorrem conflitos interdepartamentais (ex. entre o pessoal de exportação e de produção), chegamos a acordos mutuamente satisfatórios.

C4. Funcionários da unidade de exportação e aqueles de outros departamentos (ex. engenharia) ajudam-se uns aos outros.

C5. Nessa empresa, há um senso de trabalho em equipe que vai até 0 chão de fábrica (até a base da hierarquia).

C6. Na nossa empresa há um forte relacionamento de colaboração entre o pessoal de exportação e produção.

C7. As diferentes áreas funcionais desta empresa trabalham juntas na mesma direção.

Item 56 de Cadogan et al. (1999) Validado

Item 57 de Cadogan et al. (1999) Validado

Item 62 de Cadogan et al. (1999) Validado

Item 63 de Cadogan et al. (1999) Validado

Item 64 de Cadogan et al. (1999) Validado

de amostras menores, estudos em uma única indústria, eliminando efeitos de variáveis externas que possam influenciar de formas diversas a cada setor industrial, entre outras oportunidades.

No Brasil, os estudos sobre orientação para mercado, em ambiente doméstico, já se en contram em fase madura, mas praticamenteinexistem em ambientes internacionais. Esta pesquisa oferece uma escal a confiável para mensurar o grau de orientação para mercado externo das empresas exportadoras, o que pode contribuir para impulsionar os estudos nesta área.

Nesse sentido, dois temas apresentam-se como potenciais áreas de estudo no tema orientação para mercado externo. 0 primeiro é o estudo das conseqüências da orientação para mercado externo, principal mente no tocante à performance organizacional. 0 segundo tema diz respeito à possibilidade de estudos comparativos entre diferentes indústrias exportadoras brasileiras e à verificação de possíveis efeitos de moderadores sobre a relação orientação para mercado externo e performance exportadora, tais como câmbio, taxa de juros, turbulência do mercado, entre outras. Pesquisas nessa área podem contribuir de forma significativa na formação de um modelo exportador brasileiro.

\section{REFERÊNCIAS}

AKYOL, A.; AKEHURST, G. An investigation of export performance variations related to corporate export market orientation. European business Review, v. 15, n. 1, p. 5-19, 2003.

BAGOZZI, R. P. Evaluating structural equations models with unobservable variables and measurement error: a comment. Journal of M arketing Research, p. $375-381,1981$.
BAGOZZI, R. P. Structural equations models in marketing research: basic principles. In: BAGOZZI, R. P. Principles of M arketing Research. Cambridge: Blackwell, p. 317-385, 1994.

CADOGAN, J. W.; DIAMANTOPOULOS, A. Narver and Slater, Kohli and Jaworski and the market orientation construct: integration and internalization. Journal of Strategic M arketing, v. 3. p. 41-60, 1995.

CADOGAN, J. W.; DIAMANTO POULOS, A.; MORTANGES, C. P. A measure of export market orientation: scale development and cross-cultural validation. Journal of International Business Studies, v. 30, n. 4, p. 689707, 1999.

CADOGAN, J. W.; DIAMANTOPOULOS, A.; SIGUAW, J. A. Export market-oriented activities: their antecedents and performance consequences. Journal of International Business Studies, v. 33, n. 3, p. 615-626, 2002.

CONFEDERAÇÃO NACIONAL DA INDÚSTRIA. Disponível em: http:// www.brazilexport4.com Acesso em: out. 2005.

DIAMANTOPOULOS, A.; CADOGAN, J. W. Internationalizing the market orientation construct: an in-depth interview approach. Journal of Strategic Marketing, v. 4, p. 23-52, 1996.

DIAMANTOPOULOS, A.; SOUCHON, A. L. A conceptual framework of export market information use: key issues and research propositions. Journal of International Marketing, v. 4, n. 3, p. 49-71, 1996.

FARREL, M. A. Developing a market-oriented learning organization. Australian Journal of Management, v. 25, n. 2, p. 201-222, 2000.

FORNELL, C.; LARCKER, D. Evaluating structural equation models with unobservable variables with measurement error. Journal of Marketing Research, v. 18, n. 3, p. 39-50, Fev. 1981.

GARRIDO, I. L.; LARENTIS, F.; SLONGO, L. A. Validation of a scale to measure export market orientation in Brazilian export companies. BALAS Annual Conference. Lima: 2006. 
GARVER, M. S.; MENTZER, J. T. Logistics research methods: employing structural equation modeling to test for construct validity. Journal of Business Logistics, v. 20, n. 1, p. 33-57, 1999.

HAIR JR., J. F.; ANDERSO N, R. E.; TATHAM, R. L.; BLACK, W. C. Análise multivariada de dados. 5a Ed. Porto Alegre: Bookmann, 2005.

KLINE, R. B. Principle and Practice of Structural Equation M odeling. New York: The Guilford Press. 1998.

KOHLI, A. K.; JAWORSKI, B. J; KUMAR, A. MARKOR: A measure of market orientation. Journal of Marketing Research, v. 30, n. 4, p. 467-477, 1993

KOHLI, A. K.; JAW ORSKI, B. J. Market orientation: the construct, research propositions, and managerial implications. Journal of Marketing, v. 54, n. 2, p. 1-18, 1990

LUKAS, B. A.; FERREL, O. C. The effect of market orientation on product innovation. Academy of Marketing Science Journal, v. 28, n. 2, p. 239-247, 2000.
MACERA, A. P.; URDAN, A. T. O rientação para o mercado externo: teste de um modelo no Brasil e sua aplicação a uma amostra de empresas exportadoras brasileiras. Revista de Administração Contemporânea, v. 8, n. 2, p. 95-115, abr./jun. 2004.

MALHOTRA, N. K. Pesquisa de Marketing: uma orientação aplicada. 3a Ed. Porto Alegre: Bookman, 2004.

MATSUNO, K.; MENTZER, J. T.; RENTZ, J. O. A refinement and validation of the Markor scale. Journal of the Academy of Marketing Science, $v$. 28 , n. 4, p. 527-539, 2000a.

MATSUNO, K.; MENTZER, J. T.; RENTZ, J. O. The effects of strategy type on the market orientation-performance relationship. Journal of M arketing. v. 64 , n. 4, p. 1-16, 2000b.

NARVER, J. C.; SLATER, S. F. The effect of a market orientation on business profitability. Journal of Marketing, v. 54, n. 4, p. 20-35, 1990.

ZOU, S.; TAYLOR, C. R.; OSLAND, G. E. The Experf scale: a cross-national generalized export performance measure. Journal of International M arketing, v. 6, n. 3, p. 10, 1998.

\section{Artigo recebido em 04.05.2007. A provado em 20.08.2007.}

\section{Ivan Lapuente G arrido}

Doutor em Administração de Empresas pela Universidade Federal do Rio Grande do Sul. Interesses de pesquisa nas áreas de internacional ização de empresas, marketing internacional e estratégias competitivas internacionais.

E-mail: igarrido@unisinos.br

Endereço: Rua Luiz Ebling, 205-06, São Leopoldo - RS, 93042-640. 